\title{
Hadroproduction in FLUKA and Geant4: agreement with data?
}

\section{A. Bolshakova* (for the HARP-CDP group')}

Joint Institute for Nuclear Research

E-mail: nstanusun.jinr.ru

\begin{abstract}
Monte Carlo programs such as FLUKA and Geant4 have models of the production of secondary hadrons in the interactions of few $\mathrm{GeV} / \mathrm{c}$ protons and charged pions with nuclei implemented. The comprehensive and precise hadroproduction data published by the HARP-CDP group permit a critical comparison of data with modelling. Overall production cross-sections are reasonably well reproduced, within factors of two. In more detail, there are areas with poor agreement that are unsatisfactory and call for modelling improvements. Overall, the current FLUKA simulation fares better than the current Geant4 simulation.
\end{abstract}

35th International Conference of High Energy Physics - ICHEP2010,

July 22-28, 2010

Paris France

\footnotetext{
${ }^{*}$ Speaker.

${ }^{\dagger}$ The members of the HARP-CDP group are: A. Bolshakova, I. Boyko, G. Chelkov, D. Dedovich, A. Elagin, D. Emelyanov, M. Gostkin, A. Guskov, Z. Kroumchtein, Yu. Nefedov, K. Nikolaev and A. Zhemchugov from the Joint Institute for Nuclear Research, Dubna, Russian Federation; F. Dydak and J. Wotschack from CERN, Geneva, Switzerland; A. De Min from the Politecnico di Milano and INFN, Sezione di Milano-Bicocca, Milan, Italy; V. Ammosov, V. Gapienko, V. Koreshev, A. Semak, Yu. Sviridov, E. Usenko and V. Zaets from the Institute of High Energy Physics, Protvino, Russian Federation.
} 

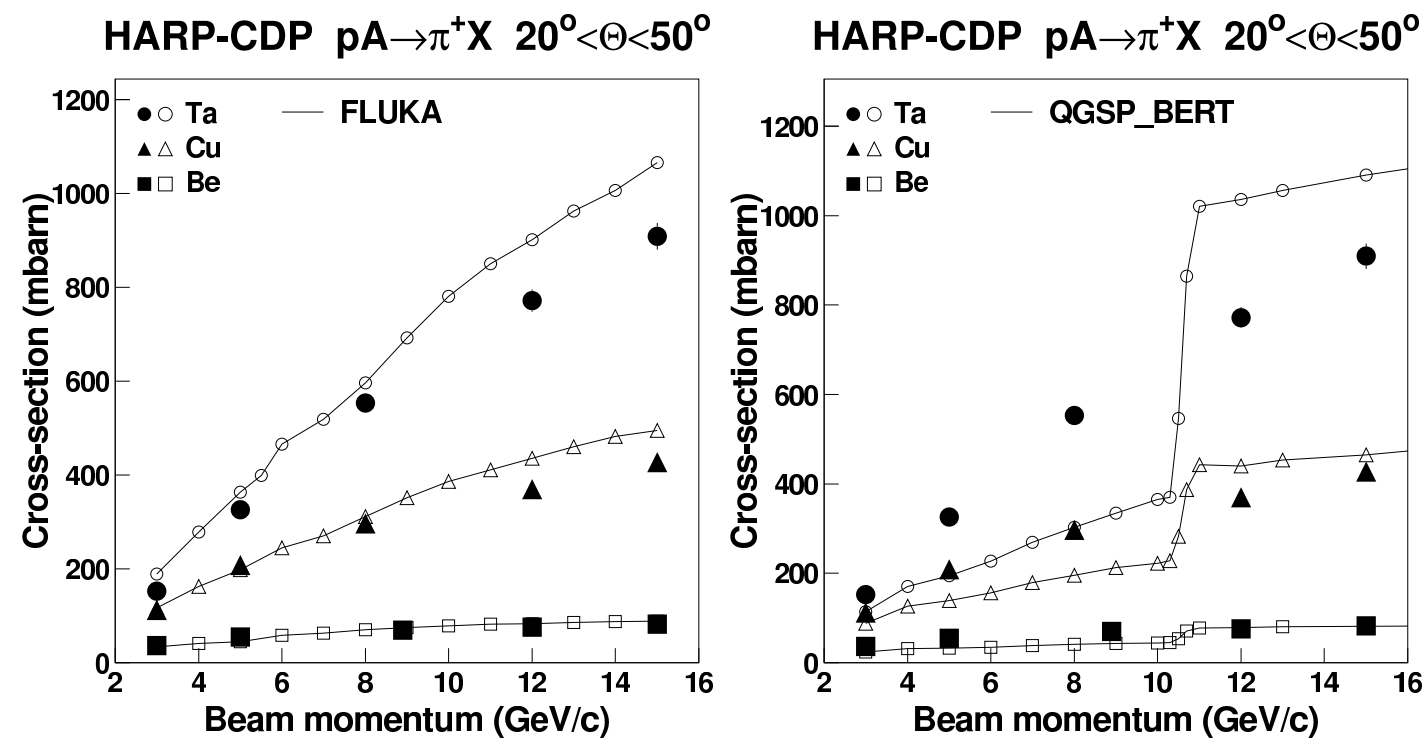

Figure 1: Comparison of measured inclusive $\pi^{+}$production cross-sections by protons on $\mathrm{Be}, \mathrm{Cu}$ and $\mathrm{Ta}$ nuclei (black symbols), in the intermediate-angle region, with FLUKA and Geant4 simulations (open symbols).

The HARP experiment took data at the CERN Proton Synchrotron in 2001 and 2002.

Several papers reported on the measurement of inclusive cross-sections of large-angle production (polar angle in the range $20^{\circ}<\Theta<125^{\circ}$ ) of secondary protons and charged pions, in the interactions with $5 \% \lambda_{\text {int }}$ beryllium, copper, tantalum and lead targets of protons and pions with beam momenta of $\pm 3.0, \pm 5.0, \pm 8.0$ ( +8.9 for beryllium), \pm 12.0 , and $\pm 15.0 \mathrm{GeV} / c$ [1]-5].

In this paper, we report on comparisons of hadroproduction data with simulations by the FLUKA and Geant4 Monte Carlo simulation programs. An example is shown in Fig. 1. It shows comparisons with FLUKA and Geant4 (we used the program versions FLUKA 2008.3c with default settings and Geant 4.9.3) simulations of measured inclusive $\pi^{+}$production cross-sections by protons on $\mathrm{Be}, \mathrm{Cu}$ and Ta nuclei. For final-state $\pi^{+}$'s, comparisons are given in the intermediateangle region $20^{\circ}<\Theta<50^{\circ}$ and in the momentum region $0.10<p_{\mathrm{T}}<0.72 \mathrm{GeV} / c$. The full set of comparisons can be found in Ref. [6]. In this parameter range, FLUKA simulations agree within $30 \%$ with our data while discrepancies of up to a factor of two are seen when comparing our data with Geant4 QGSP_BERT simulations.

\section{References}

[1] A. Bolshakova et al., Eur. Phys. J. C62 ( 2009) 293

[2] A. Bolshakova et al., Eur. Phys. J. C62 ( 2009) 697

[3] A. Bolshakova et al., Eur. Phys. J. C63 (20 09) 549

[4] A. Bolshakova et al., Eur. Phys. J. C64 (2009) 181

[5] A. Bolshakova et al., Eur. Phys. J. C66 (2010) 57

[6] A. Bolshakova et al., HARP-CDP hadroproduction data: Comparison with FLUKA and GEANT4 simulations, CERN-PH-EP-2010-017, arXiv:1006.3429, accepted for publication in Eur. Phys. J. C 